Revue des patrimoines

\title{
Paysage, architecture rurale, territoire : de la prise de conscience patrimoniale à la protection
}

\section{Arlette Auduc}

\section{OpenEdition}

Journals

Édition électronique

URL : http://journals.openedition.org/insitu/2737

DOI : 10.4000/insitu.2737

ISSN : 1630-7305

\section{Éditeur}

Ministère de la culture

Référence électronique

Arlette Auduc, «Paysage, architecture rurale, territoire : de la prise de conscience patrimoniale à la protection», In Situ [En ligne], 7 | 2006, mis en ligne le 11 avril 2012, consulté le 30 avril 2019. URL : http://journals.openedition.org/insitu/2737 ; DOI : 10.4000/insitu.2737

Ce document a été généré automatiquement le 30 avril 2019

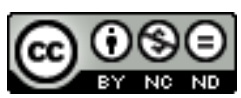

In Situ Revues des patrimoines est mis à disposition selon les termes de la licence Creative Commons Attribution - Pas d'Utilisation Commerciale - Pas de Modification 4.0 International. 


\title{
Paysage, architecture rurale, territoire: de la prise de conscience patrimoniale à la protection
}

\author{
Arlette Auduc
}

1 Depuis la Révolution la protection du patrimoine en France s'est confondue avec celle des Monuments historiques, édifices exceptionnels, "d'intérêt national pour l'histoire ou pour l'art ", comme les définissait la loi de 1887. Elle correspondait à la prédominance des valeurs esthétiques et à une lecture des œuvres d'art s'identifiant à celle de l'histoire de la civilisation occidentale comme le souligne André Chastel dans les Lieux de mémoire ${ }^{1}$.

2 Au tournant $d u X X^{e}$ siècle, l'élargissement de l'intérêt en matière d'architecture s'accompagne d'une vision plus globale du monument qui est appréhendé dans son environnement : d'abord le plus proche, le jardin, puis le site dans lequel il est édifié. Ce dernier ne tarde pas à s'émanciper du monument devenant un centre d'intérêt à part entière et un « objet » à protéger en tant que tel.

\section{La protection des jardins et des sites avant la Première Guerre mondiale}

3 Il a fallu longtemps pour que les architectes et les historiens de l'art qui siègent à la Commission des Monuments historiques s'intéressent à l'art des jardins et que ceux-ci soient considérés pour leur intérêt propre et non comme le simple écrin d'une architecture seule digne d'attention. A la fin du $\mathrm{XIX}^{\mathrm{e}}$ et au début $\mathrm{du} \mathrm{XX}^{\mathrm{e}}$ siècle la commission s'ouvre à la notion de jardins historiques à l'occasion de la redécouverte de Le Nôtre, alors qu'une nouvelle génération de jardiniers-paysagistes répond, par ses créations, à une demande de plus en plus forte d'une riche clientèle.

4 Longtemps, la protection n'a concerné que le bâtiment. Lorsque celui-ci était entouré d'un jardin, la question de son propre classement ne se posait pas, tout comme celle de 
son entretien ou de sa restauration, et on n'envisageait pas de protéger un jardin en dehors du bâti qu'il entourait. La première proposition de classement faite à la Commission des Monuments historiques date du 22 juillet 1892. Elle concerne le jardin de l'ancien évêché de Castres et émane du maire de la commune. Le rapporteur, l'inspecteur général Selmersheim, l'attribue à Jules Hardouin-Mansart, et non à Le Nôtre comme il était admis jusque-là. Mais la Commission « voit des inconvénients à entrer dans cette voie nouvelle et écarte la proposition dont il s'agit $»^{2}\left(\right.$ fig. $\left.\mathbf{n}^{\circ} \mathbf{1}\right)$.

Figure 1

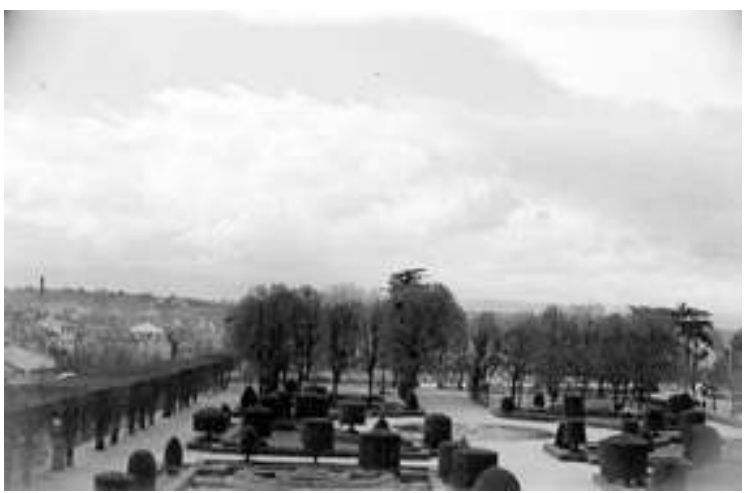

Le jardin de l'ancien évêché de Castres au début des années 1960

Phot. R. Hyvert, 1960-1963. Archives photographiques. Médiathèque de l'architecture et du patrimoine (c) $\mathrm{CMN}$

5 Pourtant un intérêt nouveau pour le végétal se fait jour et, peu à peu, le jardin intéresse pour lui-même. Le 24 février 1910, à l'occasion d'une visite à Chartres d'une délégation de la Commission, celle-ci « exprime le désir de voir maintenir, autant que possible, le jardin dans son état rustique actuel, sa transformation en square serait d'un effet déplorable et supprimerait beaucoup du charme qu'il possède actuellement ${ }^{3} »$. On ne saurait tirer des conclusions trop générales de ces cas et de quelques autres, si ce n'est qu'une nouvelle sensibilité apparaît qui ne limite plus son appréciation esthétique au seul monument indépendamment de son environnement et qui, de ce fait, est de plus en plus sensible à la qualité de celui-ci. Une préoccupation nouvelle de préservation des végétaux apparait qui accompagne une première réflexion sur la restauration des jardins. Reste évidemment un important point de doctrine: appartient-il au service des Monuments historiques de classer un jardin? En d'autres termes, un jardin est-il une œuvre d'art au même titre qu'un monument ou bien est-il un ensemble naturel dont l'appréciation relève d'autres critères ? Le débat est simplement amorcé à la séance du 17 juillet 1914 lors de l'examen de la demande de la ville de Dijon de classer son parc. Cette fois, la commission émet un avis favorable au classement. Il faudra pourtant attendre l'après-guerre pour que s'approfondisse la réflexion sur les grands domaines, comme les parcs de Saint-Cloud ou de Versailles, à propos desquels les discussions ne portent, jusque-là, que sur l'entretien des allées, des bassins ou de la statuaire.

Cet élargissement des débats est à mettre en relation avec une sensibilité nouvelle à la Nature dont les prémisses sont anciennes. Ce phénomène est aujourd'hui bien connu grâce aux travaux des historiens du paysage ${ }^{4}$ qui ont montré combien il était lié au mouvement artistique et notamment à l'histoire de la peinture. C'est le 29 novembre 1850 que la Commission examine "une réclamation adressée au Ministre par un certain nombre de peintres paysagistes au sujet d'un projet conçu par l'administration des forêts 
d'abattre les futaies du Bas-Bréau », en forêt de Fontainebleau, lieu de prédilection des peintres de l'école de Barbizon. L'Académie des Beaux-Arts est aussi saisie et le rapporteur pense « que la Commission doit intervenir en faveur de la conservation de ces arbres qui sont la meilleure école de paysage que les peintres puissent trouver dans notre pays $»^{5}$. On sait que ces paysages furent protégés en 1853 (fig. $\mathbf{n}^{\circ}$ 2).

Figure 2

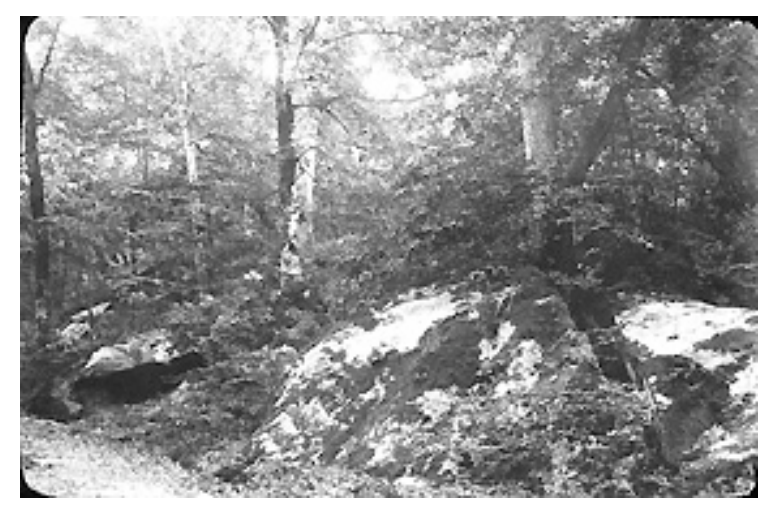

La gorge aux loups. Forêt de Fontainebleau

Phot. Touring Club de France, n. d. Archives photographiques. Médiathèque de l'architecture et du patrimoine (C) $\mathrm{CMN}$

7 Par la suite cette sensibilité paysagère s'élargit sous l'action de facteurs très divers, au premier rang desquels l'industrialisation du pays qui provoque la destruction de certains paysages, notamment en montagne où certaines vallées sont défigurées par la construction de barrages et de lignes électriques. Il y a dans le mouvement en faveur de la protection des sites une dimension anti-industrielle qui se manifeste par des attaques contre «les ingénieurs », contre la science et le progrès et par la nostalgie d'une France rurale que l'on veut croire idyllique. En même temps, l'élévation du niveau de vie, le développement de nouveaux moyens de locomotion permettent de multiplier les voyages et le tourisme. La préservation des paysages pittoresques devient alors une revendication esthétique mais aussi économique. Et cette revendication est le fait d'acteurs nouveaux, recrutés dans d'autres milieux sociaux que les membres des sociétés savantes traditionnelles du XIX siècle. Des associations se créent, qui mènent des campagnes dynamiques, utilisent des moyens de pression modernes et, par une action extrêmement volontariste, donnent naissance à un mouvement d'opinion qui presse le gouvernement de légiférer. On connaît l'activité de la Société pour la protection des paysages de France et celle du Touring Club de France qui mobilise les premiers touristes aisés (fig. $\mathbf{n}^{\circ} \mathbf{3}$ ). 
Figure 3

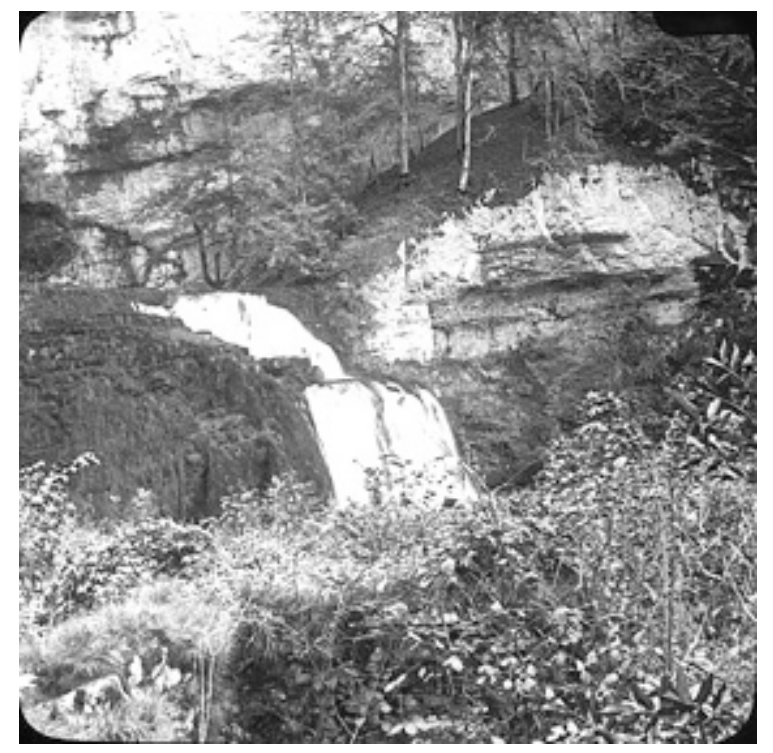

Source du Lison sauvée par l'action des associations mobilisées contre son captage afin de produire de l'électricité

Phot. J. Jablonski, 1937 (TCF). Archives photographiques. Médiathèque de l'architecture et du patrimoine (c) $\mathrm{CMN}$

Dès 1905, anticipant l'action gouvernementale, le Touring Club met en place dans les départements des Comités des sites et monuments qui associent l'appel à l'opinion publique à l'intervention directe ${ }^{6}$. Dans une note de présentation de ces comités, TCF, Revue mensuelle, de janvier 1907, explique comment obtenir le classement massif des sites à protéger : «On peut craindre des résistances devant l'afflux des demandes. Il faut que l'opinion publique réclame partout ces classements, que sa voix soit assez forte pour triompher des timidités administratives. Là encore, il faut intéresser le public à la conservation de ses richesses menacées, créer un courant d'opinion devant lequel toutes les résistances tombent, toutes les hésitations faiblissent ${ }^{7}$ ». C'est ce courant d'opinion, ainsi organisé, qui est à l'origine de la loi du 21 avril 1906, le premier projet ayant été présenté à la chambre des députés par Charles Beauquier, un des fondateurs de la Société pour la protection des paysages. Et même si ses instigateurs disent s'inspirer du système de protection des Monuments historiques, la pression de l'opinion publique, qui a précédé la volonté de l'Etat, rend cette loi profondément originale. Elle explique aussi, sans doute, son inefficacité. Laissant l'initiative de la protection aux commissions départementales des sites, la soumettant à l'accord des propriétaires, et laissant aux communes la direction de la procédure d'expropriation en cas de refus, sans prévoir d'aide financière de l'Etat, son application ne pouvait être que très limitée. Elle n'en marque pas moins la nécessaire prise en compte par l'Etat de nouvelles préoccupations en matière de préservation de la part d'une partie de l'opinion qui sait se donner les moyens de se faire entendre. Et le bilan, en 1914, n'est pas totalement négatif. Les commissions départementales ont commencé à dresser des propositions de classement dont certaines ont abouti : l'île de Bréhat (Côtes-du-Nord), le camp celtique de Bierre (Orne), les gorges du Diable (Haute-Savoie); Charles Beauquier demande à deux reprises, en 1909 et 1910, l'inscription au budget des Monuments historiques d'une somme de 10000 francs affectée aux sites. Malgré sa modestie, cette somme est refusée. Lors de la discussion du budget de 
1913, plusieurs députés proposent deux projets de résolution, la première «invite le gouvernement à proposer d'urgence les moyens nécessaires pour conserver la source et les gorges de la Loue dans leur beauté naturelle sous forme de parc national $»^{8}$, la seconde proposition demande au gouvernement «de prendre des mesures pour sauvegarder le pittoresque de la vallée du Queyras menacée par un projet de captation du Guil »'. Mais ces propositions n'aboutissent pas et il faudra attendre l'entre-deux-guerres pour qu'on tire les conséquences de cette situation.

Pourtant, de 1887 à 1914, on a assisté à un considérable élargissement de la notion de Monuments historiques. L'attachement aux témoignages du passé s'étend à des " objets » de plus en plus variés, appartenant à des époques de plus en plus diverses, d'un intérêt qui n'est plus seulement national. Plus, le goût du pittoresque amène à protéger, au-delà des bâtiments, des espaces naturels, des sites, qui témoignent aussi, à leur manière, d'une culture et d'une société menacées de disparition. Et cet élargissement n'est que rarement dû à l'initiative de l'Etat et il se poursuit durant l'entre-deux-guerres.

\section{L'entre-deux-guerres}

\section{Les jardins}

Les séances dans lesquelles sont examinés des dossiers de jardins se multiplient, même s'il ne s'agit que des plus prestigieux d'entre eux, suivant une démarche qui avait été celle du classement des monuments, passant progressivement d'un nombre limité d'édifices types à des constructions de plus en plus variées. En matière de jardins, le modèle reste le jardin ordonnancé, «à la française", plus pure production du génie national, d'où l'importance accordée aux "lignes", aux "proportions", à l'« ordonnance », à la « composition ». Ainsi, le 14 décembre 1923, la Commission se prononce en faveur du classement du château de Sceaux avec " l'ensemble du décor sylvestre et floral du parc ${ }^{10}$ " dessiné par Le Nôtre. Le jardin reste encore, bien souvent, un décor, même si l'on prend soin de préciser qu'il est inclus dans le classement d'un ensemble architectural dont il fait partie : à Reims lorsque est classé l'Hospice général (ancien collège des Jésuites), on y adjoint « le jardin (ancienne cour), y compris les ceps de vigne palissés sur les façades ${ }^{11}$, ce qui témoigne d'une conception élargie du jardin. Certains sont d'ailleurs classés indépendamment d'un monument, du fait de leur intérêt historique, ainsi «le potager de La Quintinie, à Versailles, dit Potager du Roi, servant aujourd'hui de jardin à l'Ecole d'Horticulture ${ }^{12} »$.

Si le classement de jardins «historiques" ne semble plus soulever d'objections, la question reste posée des critères qui doivent présider à cette distinction et surtout de ceux qui doivent guider leur préservation et leur restauration.

Très vite pourtant, les membres de la commission sont amenés à s'intéresser à d'autres types de jardins lorsqu'ils participent du cadre de monuments de premier ordre : le 14 mai 1923, une délégation, en visite à Versailles, se préoccupe du Petit Trianon: «La commission insiste principalement sur l'absolue nécessité de rendre son aspect traditionnel aux jardins. Le rétablissement de la rivière, complètement desséchée en certains points [...], la réfection des ponteaux qui la traversent s'imposent de toute urgence $^{13}$ ». C'est que ces jardins, négligés pendant des siècles, sont souvent en très mauvais état, lorsqu'il n'ont pas été dénaturés par des transformations malencontreuses. Comme pour les monuments au milieu du XIX ${ }^{e}$ siècle, il est nécessaire d'élaborer une 
pratique de restauration (la doctrine est généralement de retrouver l'état d'origine) par un personnel (les architectes en chef des Monuments historiques) qui n'a pas reçu de formation pour ce faire. La restauration d'un jardin étant désormais conçue comme un projet spécifique, il est nécessaire de faire appel à de nouveaux types de professionnels, plus habitués jusque-là à créer des œuvres nouvelles pour les propriétaires privés.

Ces questions se posent à une grande échelle lors de la restauration du parc de Versailles, en 1924. Des moyens financiers relativement importants y sont destinés par la donation Rockefeller ${ }^{14}$. Mais l'architecte en chef des Monuments historiques Chaussemiche semble peu intéressé par l'opération et en octobre 1924, l'administration des Monuments historiques s'émeut des violentes critiques déclenchées dans la presse par des coupes importantes pratiquées dans les parcs de Versailles et de Trianon par des jardiniers laissés à eux-mêmes. C'est Paul Léon qui, le premier, à cette occasion, fixe les bases d'une pratique qui assimile la restauration des jardins à celle des monuments. Désormais, la restauration d'un parc relève de la responsabilité de l'architecte en chef et son projet doit être présenté devant la Commission qui doit le valider. C'est un projet artistique au même titre qu'un projet de restauration de monument, ce que Paul Léon résume avec une certaine solennité : «Versailles doit être conservé tel que nous l'ont légué les siècles et l'œuvre de la Nature $»^{15}$. Mais les jardins nécessitent des compétences complémentaires à celles de l'architecte en chef ; il devra donc s'entourer de spécialistes. A Versailles, on fait appel à Forestier, conservateur des parcs et promenades de la Ville de Paris, en avance sur les services de l'Etat dans ce domaine, et à Mangin, conservateur des Eaux et Forêts de Seine-et-Oise, qui mettent au point un véritable "plan de gestion" du parc. C'est évidemment là un domaine totalement nouveau qui s'ouvre à la réflexion de la Commission. La spécificité des questions envisagées explique la création d'une souscommission permanente chargée des jardins ${ }^{16}$.

Dès lors, cette sous-commission reçoit chaque année un rapport sur les travaux effectués dans les parcs de Versailles et de Trianon. Signés généralement par un ingénieur des Eaux et Forêts, ils abordent les problèmes spécifiques des végétaux et, avec le service des jardins de Versailles et l'architecte en chef, élaborent un projet global de restauration du parc. La Commission des Monuments historiques émet un avis favorable «à la création à Versailles de pépinières pour les arbres d'alignement. Une pépinière devra être créée également pour les ifs et tous les arbustes en forme ${ }^{17} »$. Lorsque les propositions des techniciens sont acceptées par la sous-commission, l'architecte en chef établit le devis et assure le contrôle scientifique de la restauration, pour laquelle il utilise les méthodes de la restauration monumentale; le 12 mai 1930, le nouvel architecte en chef Bonnet annonce à la sous-commission de Versailles qu'il vient d'effectuer un voyage à Modène pour recueillir à la bibliothèque de cette ville une documentation devant lui permettre de poursuivre la restauration du hameau de Marie-Antoinette et du théâtre de Trianon. Grâce à des photographies et des plans dont il a la reproduction, il sera aussi à même de reconstituer les jardins et les pelouses entourant le hameau ${ }^{18}$. La restauration du Domaine de Versailles est désormais globale.

15 Assimilés aux monuments historiques, en tant que constructions du génie artistique des «jardiniers ", pour l'essentiel du grand siècle, les jardins dits historiques voient leur protection assurée dans le cadre de la loi du 31 décembre 1913. Il n'en est pas de même des sites pittoresques à propos desquels la loi de 1906 se révèle, comme il était prévu, peu efficace. 


\section{Les sites}

16 La loi du 14 mai 1930 sur les sites est donc rendue nécessaire par cet échec. En 1929, 459 sites ont été classés dont 119 seulement appartiennent à des propriétaires privés et seules quatre ou cinq expropriations ont eu lieu. La loi de 1913 est difficilement applicable aux sites : elle ne concerne que des unités spécifiques, bien définies et non des sites étendus, elle est aussi trop contraignante. D'où la nécessité de voter une nouvelle loi. Celle du 14 mai 1930, souple et équilibrée, prend acte de l'échec de la précédente loi décentralisatrice.

Le classement revient à l'Etat, mais la loi institue des commissions départementales des sites où sont représentées les associations locales, qui proposent les sites à protéger et qui dressent une liste supplémentaire: une commission supérieure se prononce sur les propositions de classement mais elle peut être saisie directement. Le classement est donc le résultat d'une réelle collaboration. Un classement d'office peut être prononcé contre une indemnité.

La loi instaure donc une plus grande centralisation: la commission supérieure assure l'unité doctrinale et scientifique en matière de classement.

Mais cette loi est souple : à côté des sites classés, le titre III crée des sites protégés : il s'agit de sites de plus vaste étendue dont le régime de protection et les prescriptions les concernant peuvent varier, en accord avec le propriétaire. Faut-il y voir une préfiguration de nos ZPPAUP?

20 En fait, la loi de 1930, étend aux « sites et [aux] monuments naturels » l'esprit de la loi de 1913. Son application pose cependant des problèmes d'une autre nature, dans la mesure où elle peut heurter des intérêts économiques considérables. Un maximum de garanties a donc été donné aux ministères concernés. Elles sont sans doute insuffisantes, puisque le 18 mars 1932, une circulaire ${ }^{19}$ du ministère de l'Instruction publique et des Beaux-arts aux préfets, rédigée par Paul Léon, attire leur attention sur le fonctionnement des commissions départementales des sites dont certaines « étendent à l'excès le champ de leur activité et vont jusqu'à s'arrêter aux sites les plus inattendus ». Sous le titre " Une loi à mieux connaître ", Georges Maringer, dans la Revue du Touring Club de France de février 1933, explique à son tour à ses délégués le fonctionnement de la loi dont il attribue la paternité au Touring Club ${ }^{20}$. Pour éviter des oppositions trop vives, il recommande une action en deux temps, d'abord l'inscription de l'ensemble des sites intéressants du département sur l'Inventaire, « cette mesure facile suffira à empêcher qu'il leur soit porté atteinte ; le classement n'interviendra que s'il devient nécessaire de les protéger contre des entreprises pouvant compromettre leur beauté ». Au-delà des procédures, ce sont les prescriptions à imposer dans les sites protégés qui posent question, ne serait-ce que parce qu'on ne possède aucun professionnel spécialiste de la gestion des paysages naturels. C'est en fait d'un pouvoir très large que sont dotées les autorités sur une partie du territoire, certes limitée, mais considérée comme exceptionnelle et dont la préservation est jugée nécessaire par les représentants les plus divers du corps social. Certains cependant ne tardent pas à juger que les instruments de sauvegarde du patrimoine sont trop dispersés et qu'ils gagneraient en efficacité et en cohérence en étant regroupés. C'est le sens du rapport lu en juin 1938 à la Commission supérieure des Monuments naturels et des Sites par l'Inspecteur Lestel ${ }^{21}$ qui rend compte notamment de propositions émises par M. Texier, maitre des requêtes honoraire et président de la Société pour l'Esthétique générale 
. Il demande l'attribution de nouvelles fonctions à la Commission supérieure des Sites dont la compétence devrait s'étendre à "tout ce qui est modification de la beauté naturelle de la France » : sites et monuments naturels, parcs et jardins, villages ruraux et grandes villes, étendant ainsi la notion de «beauté naturelle » au cadre bâti. Il demande ainsi « l'institution d'un Comité Supérieur de l'Esthétique qui coordonnerait les services existant déjà aux Beaux-arts, aux Travaux publics et à l'Intérieur et muni de pouvoirs efficaces ${ }^{22}$ ». En attendant l'institution de cette véritable police de l'esthétique, Texier propose de rendre le permis de construire obligatoire.

21 Cette nouvelle demande d'extension des pouvoirs de l'Etat à ce qui serait un contrôle de la qualité du cadre de vie des Français n'est que le dernier avatar de cet incessant élargissement, depuis un siècle, de la notion de monument historique, du monument-type d'une époque précise à des monuments de plus en plus variés, dans une fourchette chronologique de plus en plus large, puis à des jardins et des sites naturels, des ensembles urbains d'abord limités à l'entourage des monuments puis étendus à des quartiers entiers, ces sites naturels et urbains étant définis par leur "pittoresque ", notion qui tend à se substituer à celle d'intérêt artistique ou historique, national ou non. Il faudrait insister sur le contexte historique de cet élargissement, de la naissance du service des Monuments historiques sous un système politique qui cherchait à réaliser la réconciliation et l'unité historique de tous les Français et qui a utilisé ce service à cette fin, jusqu'à une Troisième République qui connaît des déchirements d'un autre ordre ; une autre fracture, celle de la Première Guerre mondiale a pris la place du traumatisme révolutionnaire, les monuments ne sont plus un enjeu historique ou national, la reconstruction a montré à quel point leur sauvegarde était intégrée à la politique nationale. Objet d'un large consensus, leur protection peut s'étendre à des objets ou des ensembles de nature différente, elle ne dépend plus que de possibilités budgétaires, de plus en plus réduites, et des nécessités d'aménagement et d'industrialisation d'un pays encore largement rural.

\section{Et le patrimoine rural ?}

L'architecture rurale n'a guère bénéficié de mesures de protection, tant les procédures créées pour les monuments historiques se sont révélées inadaptées. Architecture évolutive, faite pour être modifiée, reconstruite, pour s'adapter aux changements continus du monde rural, elle est bien différente du monument historique. Production non savante, la maison rurale est à l'opposé de l'œuvre d'art unique (fig. $\mathbf{n}^{\circ} 4$ ). 
Figure 4

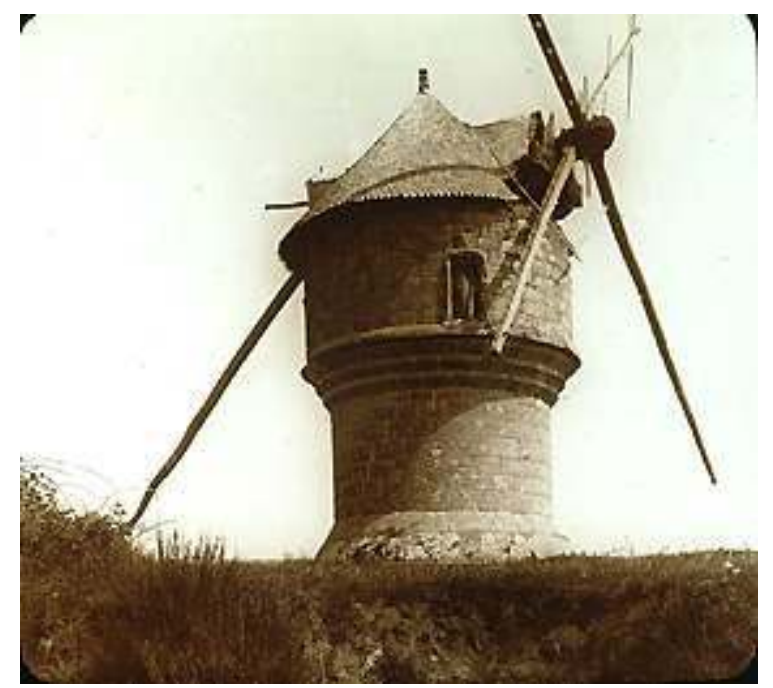

Le moulin de Crémeur (XVe s.), Guérande (Loire-Atlantique), classé MH en 1901

Phot. H. de Lestrange. Archives photographiques. Médiathèque de l'architecture et du patrimoine (c) CMN Dans la séance du 8 juillet 1937, le président (Huisman, directeur général des Beaux-Arts) signale l'intérêt que présenterait un inventaire des vieilles maisons paysannes françaises et le classement des plus remarquables. Il s'avère qu'un certain nombre d'entre elles figurent à l'Inventaire supplémentaire des monuments historiques redéfini en 1927 et que des acquisitions ont été envisagées pour la création de musées de plein air. Finalement un avis favorable est donné à la nomination de Georges-Henri Rivière comme membre de la 
première section de la commission des Monuments historiques afin d'étudier ces questions. Le 8 juillet 1938, un vœu est émis pour la protection du moulin de Chef du Bois à La Forêt-Fouesnant (Finistère), «exemple d'architecture rurale d'un intérêt exceptionnel ${ }^{24} \gg\left(\right.$ fig. $\left.n^{\circ} 5\right)$ (fig. $n^{\circ}$ 6).

Figure 5

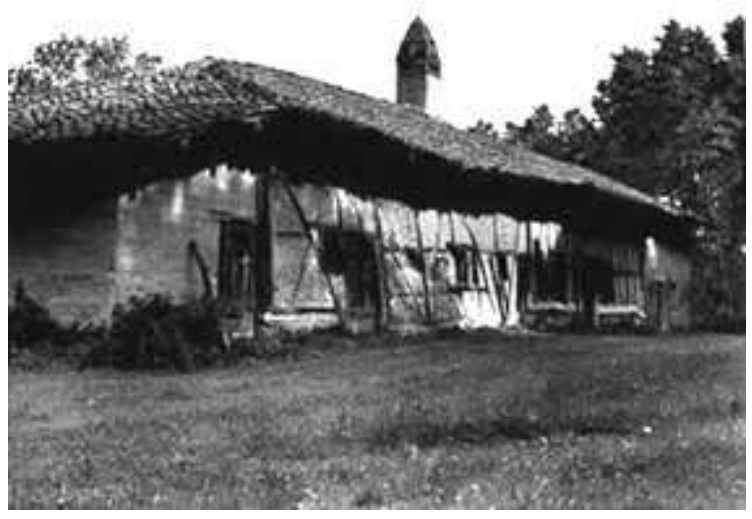

Ferme bressane à cheminée sarrasine, Saint-Romain, Romenay (Saône-et-Loire), classée MH en 1925 Centre de recherche des monuments historiques. Médiathèque de l'architecture et du patrimoine (c) $\mathrm{CMN}$

Figure 6

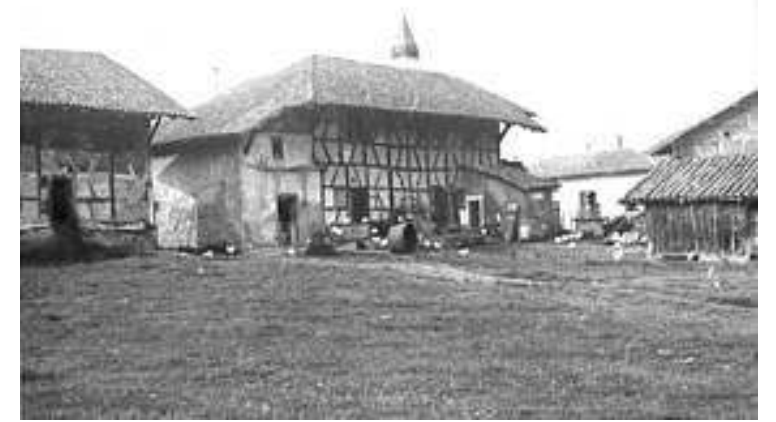

Ferme de la Forêt, à cheminée sarrasine, Courtes (Ain), classée MH en 1930

Phot. Robichon. Centre de recherche des monuments historiques. Médiathèque de l'architecture et du patrimoine (c) $\mathrm{CMN}$

Pour autant, même si les classements s'étendent à des monuments de plus en plus divers, il ne s'agit là que de l'extension de la notion traditionnelle de Monuments historiques.

\section{L'après-guerre}

C'est avec l'Inventaire général, créé en 1964, qui élargit à l'architecture rurale le recensement des richesses monumentales, que s'est amorcée la réflexion autour de la protection de ce type de patrimoine, désormais mieux connu. Si la question n'est toujours 
pas réglée, ces travaux ont contribué à sensibiliser le public et les responsables locaux, et à faire le lien avec la protection du paysage et de l'environnement naturel. La réflexion sur la protection quitte l'objet pour s'intéresser au territoire. Le problème est que la législation de protection traditionnelle, issue de la réflexion sur le monument (historique ou naturel) se révèle ici peu adéquate.

La loi sur les sites de 1930, qui concerne uniquement les paysages exceptionnels, appliquait aux monuments naturels la même conception monumentale que celle de 1913. Mais lorsqu'il s'agit d'un paysage habité, il est artificiel de séparer le bâti du paysage, car il en fait partie, le structure et l'anime. Si l'on admet cette conception globale, il faut admettre aussi que la protection de l'architecture rurale doit s'exercer de manière continue et à grande échelle, en étant associée à la protection du paysage tout entier, voire à la mise en valeur du territoire à laquelle on applique aujourd'hui le concept de développement durable.

D'autres types de protection sont cependant possibles, d'autres ministères ayant été, plus tôt que le nôtre, sensibles à ces préoccupations, bien éloignées des critères esthétiques d'origine... C'est le ministère de l'Equipement qui met en place dès les années soixante la réglementation sur les zones sensibles (1960). Surtout, à partir de 1967, sont créés les parcs naturels régionaux. Destinés à l'origine à protéger un patrimoine naturel fragile dans des zones habitées, leurs missions se sont élargies à la protection et à la mise en valeur de l'ensemble du patrimoine naturel et culturel dans une perspective de développement territorial.

31 Les années soixante-dix ont accéléré le phénomène, avec la création en 1977 des Conseils en architecture, urbanisme et environnement (CAUE) chargés de protéger la qualité de l'architecture et des paysages par leur action de conseil et d'information auprès des particuliers et des élus locaux. La loi sur l'architecture de 1977 est une étape importante. Elle affirme : «l'architecture est une expression de la culture. La création architecturale, la qualité des constructions, leur insertion harmonieuse dans le milieu environnant, le respect des paysages naturels ou urbains ainsi que du patrimoine sont d'intérêt public ». L'instauration des Zones de protection du patrimoine architectural et urbain (ZPPAU) en 1983, puis des Zones de protection du patrimoine architectural, urbain et paysager (ZPPAUP) en 1993, est un moment essentiel dans la prise en compte du patrimoine, ordinaire comme extraordinaire, dans son rapport avec son environnement, en créant des périmètres « intelligents » et concertés de protection du patrimoine.

D'autres espaces protégés ont été institués sous la direction du ministère de l'Environnement :

- Loi de 1976 sur la protection de la nature.

- Loi «montagne» en 1985 qui tend à préserver «les espaces, paysages et milieux caractéristiques du patrimoine naturel et culturel montagnard».

- Loi « littoral » de 1986.

- Loi « paysage » de 1993 qui prévoit le renforcement de la prise en compte du paysage dans les documents d'urbanisme, un volet paysager dans les permis de construire, des directives paysagères. Elle prévoit que les opérations d'aménagement foncier devront «veiller au respect et à la mise en valeur des milieux naturels, du patrimoine rural et des paysages ».

- Loi Barnier de 1995 qui crée des commissions départementales de l'environnement pour élaborer des inventaires des espaces naturels et des paysages dans les départements et qui s'intéresse aussi aux entrées de villes. 
- Loi d'orientation agricole de 1999 qui affirme le rôle environnemental et de gestion des territoires de l'activité agricole.

- Loi d'orientation et d'aménagement du territoire (LOADT) de juin 1999.

- Loi de solidarité et de renouvellement urbain (SRU) en novembre 2000. relation avec la montée de l'intérêt pour l'écologie dans les années soixante-dix; le bâti rural n'était considéré qu'à la marge. Un mouvement inverse se fait jour depuis une quinzaine d'années, bien analysé par les historiens du paysage. Le renouveau d'intérêt pour le monde rural, mais aussi les travaux universitaires, ont fait triompher l'idée, pourtant évidente, que nos paysages n'avaient rien de naturel, qu'ils étaient une construction humaine, donc culturelle, façonnée notamment par des millénaires d'agriculture et que ces agriculteurs et leur mode de vie devaient y être réintroduits. Dès lors, les lois de protection existantes, qu'elles relèvent d'une approche naturaliste ou culturelle, sont insuffisantes. Une action pédagogique de tous les acteurs publics, services de l'Etat, (de la culture, de l'agriculture, de l'équipement, de l'environnement, du tourisme, etc.), des collectivités, mais aussi des associations, est nécessaire pour conserver et faire vivre ce patrimoine. D'autant que cette conception globale ouvre à d'autres notions.

\section{Conclusion : d'autres patrimoines ruraux}

La valorisation et la transmission du patrimoine bâti et paysager supposent le maintien des techniques et des savoir-faire qui l'ont construit. Le recueil et l'inventaire en ont été faits par les folkloristes d'abord puis par les ethnologues. Cette recherche, qui dépasse le cadre de cette étude, a débouché sur l'élargissement de la notion du patrimoine aux divers produits du terroir. C'est l'ensemble de ces notions qui étaient englobées dans celle de patrimoine culturel rural dans le rapport d'Isaac Chiva en 1994. On est bien loin de l'attention portée au seul monument d'exception ou même au seul bâti rural, celui-ci ne pouvant se comprendre et donc se protéger qu'inséré dans un ensemble, fruit d'évolutions mais aussi en devenir, donc un patrimoine vivant pour l'étude duquel il faut tenir compte de ces deux dimensions essentielles que sont le temps et l'usage. Mais n'estce pas le cas de tous les patrimoines?

\section{ANNEXES}

\section{Chronologie des textes législatifs}

Débuts du XX $\mathrm{X}^{\mathrm{e}}$ siècle : émergence de l'idée de protection

- Loi du 21 avril 1906 : loi de protection des « monuments naturels et des sites ».

- Loi du 31 décembre 1913 : loi de protection des monuments historiques. 
Années 1930-40 : la protection des sites

- Loi du 21 juillet 1927 sur l'inventaire supplémentaire des Monuments historiques.

- Loi du 2 mai 1930 sur la protection monuments naturels et sites de caractère artistique, historique, scientifique, légendaire ou pittoresque.

- 25 février 1943: Loi sur les abords des monuments historiques instituant un périmètre de protection de $500 \mathrm{~m}$. autour d'un monument historique.

- 2 novembre 1945 : ordonnance modifiant la loi du 2 mai 1930 : elle introduit pour la première fois le mot "paysage » en remplaçant la commission des sites et monuments naturels par une commission des sites et paysages.

Années 1960 : le paysage naturel et urbain

- 1960 : Loi sur les parcs nationaux.

- 30 novembre 1961 : décret portant règlement national d'urbanisme. Introduit par l'article R 111-21 du Code de l'Urbanisme l'expression «paysages naturels ou urbains » que les opérations d'urbanisme se doivent de protéger.

- 4 août 1962 : Loi relative aux secteurs sauvegardés.

- Création de l'Inventaire général des monuments et des richesses artistiques de la France.

- 1967 : Décret interministériel sur les Parcs Naturels Régionaux.

Années 1970 : environnement, architecture

- 10 juillet 1976 : Loi sur la protection de la nature. Décrets d'application pris en 1977 instituant les études d'impact pour les grandes infrastructures et équipements. Prise en compte des paysages dans les POS. Création des réserves naturelles.

- 3 janvier 1977 : Loi sur l'architecture : «La qualité de l'architecture et du cadre de vie est d'intérêt général ». Création des CAUE.

Années 1980 : la décentralisation

- 7 janvier 1983 : Loi sur la décentralisation. Instaure les ZPPAU.

- 9 janvier 1985 : Loi « montagne ».

- 18 juillet 1985 : Loi sur les espaces naturels sensibles des départements, qui vise à préserver « la qualité des sites, paysages et milieux naturels ».

- 3 janvier 1986 : Loi « littoral».

Années 1990 : le paysage

- 8 janvier 1993 : Loi « paysage » (loi Royal) : plan d'action en faveur de la protection et de la mise en valeur du paysage : renforcement de la prise en compte du paysage dans les documents d'urbanisme, volet paysager du permis de construire, directives paysagères, instauration des ZPPAUP.

- 2 février 1995 : Loi relative au renforcement de la protection de l'environnement (loi Barnier) : commissions départementales de l'environnement, inventaire des espaces naturels et des paysages dans les départements. Amendement Dupont sur les entrées de villes.

- 16 juin 1999 : Loi d'orientation sur l'aménagement durable du territoire (LOADT, loi Voynet) : création des " pays ", des schémas collectifs de services, chartes des PNR opposables aux documents d'urbanisme.

- 9 juillet 1999 : Loi d'orientation agricole : CTE (contrats territoriaux d'exploitation), affirmation du rôle environnemental et de gestion des territoires des agriculteurs.

- 13 décembre 2000 : Loi SRU. 


\section{NOTES}

1. Chastel, André. La notion de patrimoine. Les Lieux de mémoire II, La Nation. Dir. Pierre Nora. Paris : Gallimard, 1986, p. 405-449.

2. Archives du Patrimoine, 80/15/14, séance de la commission des Monuments historiques, 22 juillet 1892.

3. Archives du Patrimoine, 80/15/14, séance de la commission des Monuments historiques, 22 juillet 1892.

4. Signalons les travaux d'Alain Roger, d'Augustin Berque ou de Serge Briffaud.

5. Archives du Patrimoine, 80/15/7, séance de la commission des Monuments historiques, 29 novembre 1850.

6. Bertho-Lavenir, Catherine. La roue et le stylo. Comment nous sommes devenus touristes. Paris : Odile Jacob, 1999.

7. Cité par Catherine Bertho-Lavenir. La roue et le stylo. Comment nous sommes devenus touristes. Paris : Odile Jacob, 1999.

8. Journal officiel. Débats parlementaires, Chambre des députés, séance du 6 décembre 1912.

9. Journal officiel. Débats parlementaires, Chambre des députés, séance du 6 décembre 1912.

10. Archives du Patrimoine, 80/15/25, séance de la commission des Monuments historiques, 20 juillet 1923.

11. Archives du Patrimoine, $80 / 15 / 29$, séance de la commission des Monuments historiques, 7 juillet 1933.

12. Archives du Patrimoine, $80 / 15 / 26$, séance de la commission des Monuments historiques, 18 décembre 1925.

13. Archives du Patrimoine, 80/15/25, séance de la commission des Monuments historiques, 14 mai 1923.

14. Archives du Patrimoine, $80 / 15 / 25$, séance de la commission des Monuments historiques, 14 mai 1923. Dans sa séance du 10 juillet 1924, la commission des Monuments historiques décide de la répartition des 9 millions de francs de la donation Rockefeller destinés à la restauration de Versailles. 5 millions vont à la «remise en état des parcs et jardins", dont 3 millions pour la réfection des treillages et l'élagage des arbres et 2 millions pour la réfection des charmilles, plantations et travaux divers de remise en état dans les parcs et jardins de Versailles et de Trianon.

15. Dans sa séance du 10 juillet 1924, la commission des Monuments historiques décide de la répartition des 9 millions de francs de la donation Rockfeller destinés à la restauration de Versailles. 5 millions vont à la « remise en état des parcs et jardins ", dont 3 millions pour la réfection des treillages et l'élagage des arbres et 2 millions pour la réfection des charmilles, plantations et travaux divers de remise en état dans les parcs et jardins de Versailles et de Trianon.

16. Séance de la commission des Monuments historiques, 24 octobre 1924.

17. Archives du Patrimoine, $80 / 15 / 29$.

18. Archives du Patrimoine, $80 / 15 / 27$, séance de la commission des Monuments historiques, 12 mai 1930.

19. Archives du Patrimoine, $80 / 15 / 27$, séance de la commission des Monuments historiques, 12 mai 1930. Circulaire du ministère de l'Instruction publique et des Beaux-arts, 18 mars 1932.

20. Maringer, Georges. Une loi à mieux connaître. Revue du Touring Club de France, février 1933, n 459, p. 39-40. 
21. Archives du Patrimoine, $80 / 15 / 27$. Rapport à la commission supérieure des Monuments naturels et des Sites par M. Lestel, inspecteur des Monuments naturels et des sites, sur l'amélioration de la loi de protection des Monuments naturels et des Sites, juin 1938.

22. Archives du Patrimoine, 80/15/27. Rapport du Cabinet du ministre de l'Education nationale sur les décrets préparés par M. Texier, 28 juin 1938.

23. Archives du Patrimoine, $80 / 15 / 29$.

24. Archives du Patrimoine, $80 / 15 / 29$.

\section{RÉSUMÉS}

Longtemps, la protection du patrimoine, en France, s'est confondue avec celle des Monuments historiques, édifices exceptionnels, "d'intérêt national pour l'histoire ou pour l'art », comme les définissait la loi de 1887. Pourtant de 1887 à 1914, on a assisté à un considérable élargissement de la notion de Monuments historiques. L'attachement aux témoignages du passé s'étend à des «objets » de plus en plus variés, d'époques de plus en plus larges, d'un intérêt qui n'est plus seulement national. L'architecture rurale n'a guère bénéficié de ces mesures de protection, tant les procédures créées pour les Monuments historiques se sont révélées inadéquates. Architecture évolutive, faite pour être modifiée, reconstruite, pour s'adapter aux changements continus du monde rural, elle est bien différente du monument historique. Production non savante, la maison rurale est à l'opposé de l'œuvre d'art unique. Quelques mesures de classement ont cependant été prises et surtout des inscriptions à l'Inventaire supplémentaire des Monuments historiques d'exemples d'architectures rurales choisis surtout pour leur intérêt ethnologique sous l'impulsion de Georges-Henri Rivière. C'est avec l'Inventaire général élargissant à l'architecture rurale le recensement des richesses monumentales que s'est amorcée la réflexion autour de la protection de ce type de patrimoine, désormais mieux connu. Aujourd'hui, la réflexion sur la protection quitte l'objet pour s'intéresser au territoire. Mais lorsqu'il s'agit d'un paysage habité, il est artificiel de séparer le bâti du paysage, car il en fait partie, le structure et l'anime. Si l'on admet cette conception globale, il faut admettre aussi que la protection de l'architecture rurale doit s'exercer de manière continue et à grande échelle, en étant associée à la protection du paysage tout entier, voire à la mise en valeur du territoire. D'autres types de protection que celles issues des Monuments historiques sont alors possibles, d'autres ministères ayant été, plus tôt que le nôtre, sensibles à ces préoccupations, bien éloignées des critères esthétiques d'origine.

For a long time, heritage protection in France meant the protection of historic monuments, exceptional buildings "of national interest for history or for art", as the law of 1887 formulated it. From 1887 to 1914, however, the notion of what a historic monument might be was considerably broadened. Attachment to significant traces of the built past began to encompass 'objects' of increasing diversity and from different historical periods and 'national' interest was no longer the exclusive yardstick. Rural architecture benefited little from measures of protection, however, the procedures elaborated for historic monuments being ill suited to these new objects. Rural architecture is an architecture which evolves. Constantly modified, rebuilt and adapted to continuous changes in rural life, it is very different from the historic monument. The rural dwelling is not a book-inspired production and may indeed be seen as the antithesis of the unique work of art. Nonetheless some examples of rural architecture have been given protection ('classement' or, more frequently, 'inscription'), examples often selected for their 
ethnological values, under the influence of Georges-Henri-Rivière. For forty years, the services of the 'Inventaire général' have been studying rural architecture in their research programmes and it is around such inventory and survey work that, inspired by better understanding, new ideas have emerged as to how this type of heritage can be protected. Today, these ideas focus not so much on the individual built object as on the territory to which it belongs. When this territory is an inhabited one, it is artificial to separate the building from its landscape. It is part of this landscape, structuring it and giving it life. If this more holistic approach is adopted, it follows that the protection of rural architecture must be capable of continuity and evolution on a large scale, associated with the preservation of the landscape as a whole and the valorisation of the territory. Forms of protection different from those based on the notion of the historic monument are possible. Before the Ministry of Culture, other ministries have already addressed this problem, sensitive to the protection of landscapes with criteria which have little to do with the aesthetic ones at the origins of monument protection.

\section{INDEX}

Mots-clés : patrimoine rural, architecture, paysage, monuments historiques, jardins, sites, protection, conservation

Keywords : rural heritage, landscape, historic monuments, gardens

\section{AUTEUR}

\section{ARLETTE AUDUC}

Chargée de mission. Direction de l'architecture et du patrimoine, bureau de la création architecturale, du paysage et du cadre de vie. arlette.auduc@culture.gouv.fr 